\title{
PRODUTIVIDADE E CRESCIMENTO DA MAMONEIRA EM PLANTIO ADENSADO SOB DIFERENTES NÍVEIS DE IRRIGAÇÃO
}

\author{
Wezer Lismar MIRANDA ${ }^{1}$; Luiz Gonsaga de CARVALHO ${ }^{2}$; Gleice Aparecida de ASSIS ${ }^{3}$; \\ Pedro Castro NETO ${ }^{4}$; Fátima Conceição REZENDE ${ }^{5}$; Adriano Valentim DIOTTO ${ }^{6}$
}

\begin{abstract}
${ }^{1}$ Professor nos cursos de Agronomia, Engenharia Ambiental e mestrado em Sustentabilidade em Recursos Hídricos, UNIVERSIDADE VALE DO RIO VERDE. E-mail: wmlismar@gmail.com.

${ }^{2}$ Departamento de Engenharia/Agrometeorologia, UNIVERSIDADE FEDERAL DE LAVRAS, E-mail: lgonsaga@deg.ufla.br.

${ }^{3}$ Departamento de Agricultura, UNIVERSIDADE FEDERAL DE UBERLÂNDIA, E-mail: gleiceufu@gmail.com.

${ }^{4}$ Departamento de Engenharia/ Agrometeorologia, UNIVERSIDADE FEDERAL DE LAVRAS, E-mail: pedrocn@deg.ufla.br.

${ }^{5}$ Departamento de Engenharia/Hidráulica, UNIVERSIDADE FEDERAL DE LAVRAS, E-mail: frezende@,deg.ufla.br.

${ }^{6}$ Departamento de Engenharia/Hidráulica, UNIVERSIDADE FEDERAL DE LAVRAS, E-mail: adriano.diotto@gmail.com.
\end{abstract}

Recebido em: 31/05/2017 - Aprovado em: 06/09/2017 - Disponibilizado em: 30/12/2017

Resumo: O anseio pela melhora da rentabilidade agrícola tem levado ao emprego de técnicas variadas que vinham sendo relegadas ao cultivo da mamoneira. Dentre estas, cita-se o adensamento e a irrigação. Assim, com o presente trabalho objetivouse avaliar características de produção e crescimento da mamoneira cultivada em espaçamento adensado e irrigada com base em diferentes níveis de tensão de água no solo. O experimento foi conduzido na área de pesquisa do setor de Agrometeorologia e Climatologia da Universidade Federal de Lavras, em Lavras - MG, no período de março a setembro de 2010. O espaçamento de plantio foi de $0,30 \mathrm{~m}$ entre plantas e $0,70 \mathrm{~m}$ entre linhas. Avaliou-se altura do caule, diâmetro de copa, peso das sementes com baga e peso das sementes sem baga. Utilizou-se o delineamento experimental de blocos casualizados, com cinco tratamentos e quatro repetições. Os tratamentos consistiram de: uma testemunha sem irrigação (T0) e da irrigação sempre que o potencial matricial a $0,20 \mathrm{~m}$ de profundidade atingiu os valores de 15 (T1), 30 (T2), 45 (T3) e $60 \mathrm{kPa}$ (T4). O tratamento T1 foi o que apresentou maior produtividade e crescimento. A produtividade deste tratamento foi de $4700 \mathrm{~kg} \mathrm{ha}^{-1}$, enquanto a da testemunha foi de $460 \mathrm{~kg} \mathrm{ha}^{-1}$.

Palavras-chave: Ricinus communis L. Safrinha. Espaçamento. Tensiômetro. Gotejamento.

\section{PRODUCTIVITY AND GROWTH OF THE CASTOR BEAN WHILE SUBJECTED TO HIGH PLANT POPULATION UNDER DIFFERENT IRRIGATION LEVELS}

\begin{abstract}
Presented as an alternative for biodiesel production without direct competition in the food market, the castor bean crop has received more attention in an attempt to improve agricultural profitability. Some techniques which had not previously been considered are now receiving increasing investigation, such as high density of plant population and irrigation. This present study aimed to evaluate castor bean yield and growth characteristics in the field with narrow row spacing, and under irrigation based on different levels of soil water tension. The research field was located in the Climatology and Agrometeorology sector, of the engineering department at the University of Lavras. The evaluated period was from March to September of 2010. The plant spacing was $0.30 \mathrm{~m}$ between plants and $0.70 \mathrm{~m}$ between rows. The stem height, plant diameter, seed weight with and without berry were evaluated. A completely randomized design with five treatments and four replications was used. Treatments were without irrigation (T0); and irrigation starting at soil matric potential values of 15 (T1), 30 (T2), 45 (T3), and $60 \mathrm{kPa}$ (T4). Treatment $\mathrm{T} 1$ presented the highest yield and growth. This treatment showed $4700 \mathrm{~kg} \mathrm{ha}^{-1}$ and the control without irrigation just $460 \mathrm{~kg} \mathrm{ha}^{-1}$.
\end{abstract}

Keywords: Ricinus communis L. Second crop. Plant population. Tensiometer. Drip irrigation.

\section{INTRODUÇÃO}

\section{A mamoneira (Ricinus communis}

L.) é uma planta oleaginosa da família das euforbiáceas, que a exemplo de outras culturas como a soja e o girassol, também tem sido utilizada para produção de biocombustíveis, mas com a vantagem de não exercer pressão significativa na concorrência com o mercado de gêneros alimentícios e de insumos para pecuária.

Ante as diversas possibilidades de uso da mamoneira, conforme Rodrigues et al. (2009) destacam, fazendo menção ao fato do óleo extraído de suas sementes 
servir de matéria prima para várias indústrias, além de seus outros produtos e coprodutos, o cultivo da mamoneira tem se disseminado para regiões que tradicionalmente tinham o agronegócio voltado para outros cultivos. Assim, passou-se a considerar a aplicação de técnicas como o plantio direto, irrigação e colheita mecanizada, empregadas em outras culturas, também ao cultivo da mamoneira.

A aplicação das referidas técnicas se mostraram tão promissoras que instituições de pesquisa passaram inclusive a desenvolver novas cultivares de mamona para tal fim. Exemplo disto pode ser visto no trabalho de Savy Filho et al. (2007), no qual apresentam a cultivar IAC-2028, destacando sua adaptação à semeadura mecanizada que associada ao seu porte baixo, pode ser indicada para arranjos populacionais de até 20.000 plantas por hectare, quando cultivada na safrinha e na dependência da disponibilidade hídrica.

Nesse sentido, Souza-Schlick et al. (2011), avaliando o desempenho dessa mesma cultivar em função do espaçamento entre fileiras e população de plantas na safrinha em Botucatu (SP), observaram maior produtividade - cerca de $2.300 \mathrm{~kg} \mathrm{ha}^{-}$ ${ }^{1}$ - nos espaçamentos de 0,45 e 0,60 m entre fileiras. Soratto et al. (2011) testando a cultivar FCA-PB, também concluíram que as maiores produtividades foram obtidas com arranjos populacionais de 55.000 e
70.000 plantas por hectare, com produtividade observada de até 4.882 e $5.171 \mathrm{~kg} \mathrm{ha}^{-1}$, respectivamente, em safra de verão.

Trabalhos semelhantes também foram desenvolvidos por Carvalho et al. (2010), Souza-Schlick et al. (2012) e Severino et al. (2006), todos indicando que o adensamento de plantio promove ganhos em produtividade.

Quanto à reposição de água, há diversos trabalhos utilizando diferentes métodos e sistemas de irrigação aplicados à mamoneira. No entanto, Freitas et al. (2010) destacam que para crescer, desenvolver e produzir satisfatoriamente, a mamoneira necessita de suprimento hídrico diferenciado nas suas fases fenológicas, o que requer manejo compatível com sua capacidade de retirada de água do solo; desta forma, o manejo e a qualidade da água da irrigação podem implicar em redução ou aumento da produtividade para a mamoneira.

Neste contexto, apesar da mamoneira ser uma planta resistente a certos níveis de déficit hídrico, Carvalho (2005) mostra que o potencial de produtividade médio para duas cultivares variaram de $1.500 \mathrm{~kg} \mathrm{ha}^{-1}$ em condições de sequeiro a $5.000 \mathrm{~kg} \mathrm{ha}^{-1}$ em condições irrigadas, o que demonstra a viabilidade técnica da irrigação para a cultura. 
Segundo Beltrão (2004), no Brasil, as áreas irrigadas com mamoneira são poucas, com alguns registros na Bahia, no Rio Grande do Sul e no Maranhão, mas atingindo mais de $6.000 \mathrm{~kg}$ de baga ha- ${ }^{-1}$, em alguns casos.

Ademais, ganhos de produtividade e maior desenvolvimento de características agronômicas da mamoneira devido a irrigação, também foram observados por Viana et al. (2009) estudando a cultivar IAC Guarani, em Fortaleza, utilizando um sistema de irrigação por gotejamento e manejo baseado na evaporação de um tanque reduzido. As mesmas vantagens são apontadas em trabalho de Barros Júnior et

\section{MATERIAL E MÉTODOS}

O experimento foi conduzido na área de pesquisa do Setor de Agrometeorologia e Climatologia da Universidade Federal de Lavras, no município de Lavras, MG, situado a altitude de $918,8 \mathrm{~m}$; latitude de $21^{\circ} 14^{\prime} \mathrm{S}$; e longitude de $45^{\circ} 00^{\prime} \mathrm{W}$, tendo como referência a Estação Climatológica Principal, pertencente a rede de observações meteorológicas de superfície do INMET Instituto Nacional de Meteorologia - no convênio UFLA/INMET.

O solo é classificado originalmente como Latossolo Vermelho Distroférrico al. (2008), testando a cultivar BRS 149, em Campina Grande, manejando a irrigação com auxílio de TDR.

No entanto, dos apontamentos feitos até aqui, uma lacuna que fica sem resposta e para a qual não se encontrou trabalhos semelhantes na literatura consultada é relacionada ao efeito da irrigação no plantio adensado da mamoneira em condições de safrinha. Assim, com o presente trabalho, objetivou-se avaliar a produtividade $\mathrm{e}$ aspectos do crescimento da mamoneira em plantio adensado no período de safrinha e irrigada com base em diferentes níveis de tensão de água no solo.

(LVdf) segundo o Sistema Brasileiro de Classificação de Solos (EMBRAPA, 2006). A curva de retenção de água no solo foi obtida para as camadas de 0 a $0,20 \mathrm{~m} ; 0,20$ a $0,40 \mathrm{~m}$; e 0,40 a $0,60 \mathrm{~m}$ de profundidade. As referidas camadas foram amostradas e, em laboratório, foram determinados os pares de tensão e umidade do solo que deram origem a curva de retenção, de acordo com o modelo proposto por Van Genuchten (1980), com auxílio do programa computacional Soil Water Retention Curve (SWRC), elaborado por Dourado Neto et al. (1990). As equações ajustadas estão apresentadas na Tabela 1. 
Tabela 1. Equações de retenção de água no solo para as três profundidades do perfil de solo na área experimental, em Lavras-MG, 2010.

\begin{tabular}{cc}
\hline Camadas (m) & Equações Ajustadas \\
\hline $0-0,20$ & $\theta=0,221+\frac{0,377}{\left[1+\left(0,228 \times\left|\psi_{m}\right|\right)^{1,879}\right]^{p, 468}}$ \\
$0,20-0,40$ & $\theta=0,230+\frac{0,374}{\left[1+\left(0,265 \times\left|\psi_{m}\right|\right)^{2,024}\right]^{p, 506}}$ \\
$0,40-0,60$ & $\theta=0,238+\frac{0,423}{\left[1+\left(1,134 \times\left|\psi_{m}\right|\right)^{1,629}\right]^{p, 386}}$
\end{tabular}

Fonte: (LIMA; SILVA, 2008; RIOS et al., 2014)

onde, $\theta$ representa a umidade atual do solo $\left(\mathrm{cm}^{3} \mathrm{~cm}^{-3}\right)$ e $\psi_{\mathrm{m}}$ o potencial matricial da água no solo $(\mathrm{kPa})$.

A classificação climática proposta por Köppen é do tipo Cwa, com inverno seco e chuvas predominantes no verão, com precipitação total média anual de $1530 \mathrm{~mm}$ e temperatura média anual de $19,4{ }^{\circ} \mathrm{C}$ (DANTAS et al., 2007).

A cultivar utilizada foi a Al Guarany 2002 que possui porte médio, variando entre 1,6 e 2,4 m de altura, ciclo de 180 dias, sementes de tamanho médio, e com produtividade média variando entre $1.000 \mathrm{e}$ $2.500 \mathrm{~kg} \mathrm{ha}^{-1}$ (BRASIL, 2010).

O preparo de solo foi realizado de forma convencional e o transplantio em campo feito em sulcos com aproximadamente $0,20 \mathrm{~m}$ de profundidade, no espaçamento de $0,30 \mathrm{~m}$ entre plantas e 0,70 m entre linhas. Destaca-se a diferença deste espaçamento em relação ao recomendado pela Coordenadoria de Assistência Técnica Integral - CATI (AMARAL, 2002), que para esta cultivar estabelece 1,5 x 1,0 m para semeadura de verão e 1 x $1 \mathrm{~m}$, em solos de baixa fertilidade e/ou semeadura de safrinha.

A adubação foi realizada com base nos resultados da análise de fertilidade do solo e atendeu às recomendações da Comissão de Fertilidade do Solo do Estado de Minas Gerais (GUIMARÃES et al., 1999).

A semeadura foi feita em janeiro de 2010, o transplantio em campo ocorreu no mês de março do mesmo ano. Para garantir o pegamento das mudas, todas as parcelas receberam a mesma lâmina de água até a diferenciação dos tratamentos que ocorreu no mês de abril. A colheita foi realizada em setembro de 2010 e as avaliações foram feitas em quatro épocas (E) entre os meses de maio e agosto. 
Foram avaliadas altura do caule em $\mathrm{cm}$, da superfície do solo até o ponto de inserção do primeiro racemo; diâmetro de copa - em cm, transversalmente a linha de plantio considerando as extremidades das folhas; peso das sementes com baga e peso das sementes sem baga.

Utilizou-se o delineamento experimental de blocos casualizados, com cinco tratamentos e quatro repetições, totalizando vinte parcelas experimentais. As parcelas foram constituídas de três linhas de plantio com dez plantas cada. Considerou-se oito plantas da linha central como úteis e as demais como bordadura. Os tratamentos consistiram de: uma testemunha sem irrigação (T0) e da aplicação de água, até a capacidade de campo, sempre que o potencial matricial a $0,20 \mathrm{~m}$ de profundidade atingiu os valores de 15 (T1), 30 (T2), 45 (T3) e 60 kPa (T4).

Para determinar os referidos níveis de retenção de água no solo, utilizou-se

\section{RESULTADOS E DISCUSSÃO}

Observou-se efeito significativo das épocas (E) de avaliação na altura das plantas ao nível de $1 \%$ de probabilidade pelo teste tensiômetros de vacuômetro digital, também instalados nas profundidades de 0,$10 ; 0,40$ e $0,60 \mathrm{~m}$ com o intuito de garantir o adequado manejo da irrigação. As leituras nos tensiômetros foram realizadas diariamente pela manhã e à tarde, com auxílio de um tensímetro de punção digital.

O sistema de irrigação utilizado foi o localizado com gotejamento em linhas laterais de polietileno e emissores autocompensantes com vazão nominal de 2,2 $\mathrm{L} \mathrm{h}^{-1}$ espaçados de $0,7 \mathrm{~m}$.

Os dados das avaliações de crescimento e produtividade foram analisados pelo programa estatístico SISVAR (FERREIRA, 2011). Para o estudo da interação entre os fatores épocas de avaliação e níveis de tensão de água no solo, foram realizadas análises de regressão para cada característica avaliada dentro de cada época de avaliação.

de F (Tabela 2), e a $10 \%$ de probabilidade para a interação entre tensões e épocas de avaliação. 
Tabela 2. Resumo da análise de variância para as características altura de planta $(\mathrm{cm})$ e diâmetro de copa $(\mathrm{cm})$ de mamoneira irrigada sob diferentes tensões, em Lavras-MG, 2010.

\begin{tabular}{lccc}
\hline \multirow{2}{*}{ FV } & GL & \multicolumn{2}{c}{ Quadrados Médios } \\
\cline { 3 - 4 } & & Altura & $\begin{array}{c}\text { Diâmetro de } \\
\text { copa }\end{array}$ \\
\hline Tensões (T) & 3 & $347,60^{\text {ns }}$ & $210,51^{\text {ns }}$ \\
Blocos & 3 & $1099,60^{* *}$ & $303,62^{\text {ns }}$ \\
Erro 1 & 9 & 219,75 & 226,64 \\
Épocas (E) & 3 & $12010,85^{* * *}$ & $1530,44^{\text {ns }}$ \\
Erro 2 & 9 & 202,28 & 883,52 \\
T X E & 9 & $84,19^{*}$ & $161,40^{\text {ns }}$ \\
Erro 3 & 27 & 40,59 & 128,11 \\
\hline CV1(\%) & & 22,05 & 21,67 \\
CV2(\%) & & 21,16 & 42,78 \\
CV3(\%) & 9,48 & 16,29 \\
\hline Média & & 67,2 & 69,5 \\
\hline ns - não significativo, ${ }^{* * *}$ significativo a 1\%,** significativo a 5\%, & significativo a 10\% de probabilidade, pelo \\
teste F. & & &
\end{tabular}

Já os diferentes níveis de tensão de água no solo, isoladamente, não apresentaram efeito significativo para a variável altura de plantas. Este efeito diverge do observado por Silva et al. (2009) em experimento que avaliou a influência de quatro diferentes lâminas de irrigação no crescimento da mamoneira, sendo que houve acréscimos nos valores de altura a medida que se aumentou a lâmina de irrigação de 294,22 mm (L1) para 382,50 mm (L2) e para 479,75 mm (L3). A maior lâmina, no valor de $679,75 \mathrm{~mm}$ (L4), provocou sintomas de anoxia.
Analisando os resultados observados para diâmetro de copa, este não apresentou efeito significativo para nenhum dos fatores propostos, possivelmente, em razão da limitação de crescimento imposta pelo espaçamento de plantio, uma vez que a parte aérea das plantas vizinhas na linha e entre linhas se tocavam desde a primeira avaliação.

Mediante $o$ efeito significativo obtido para a interação T x E para a variável altura de plantas, realizou-se $\mathrm{o}$ desdobramento da tensão dentro de cada época de avaliação conforme apresentado na Tabela 3.

Tabela 3. Resumo da análise de variância para o desdobramento de tensões de irrigação dentro de cada época, para altura das plantas, em Lavras-MG, 2010.

\begin{tabular}{ccc}
\hline FV & GL & Quadrados médios (Altura) \\
\hline Tensões: Época 1 & 3 & $5,84^{\mathrm{ns}}$ \\
Tensões: Época 2 & 3 & $10,61^{\mathrm{ns}}$ \\
Tensões: Época 3 & 3 & $240,25^{*}$ \\
Tensões: Época 4 & 3 & $343,48^{*}$ \\
\hline Erro & 27 & 40,59 \\
\hline
\end{tabular}

ns - não significativo, ${ }^{*}$ significativo a $1 \%$ de probabilidade, pelo teste $\mathrm{F}$. 
$\mathrm{Na}$ análise de variância deste desdobramento é possível observar que somente a partir da terceira avaliação obteve-se diferença significativa para a altura entre os tratamentos. Este fato pode estar relacionado com a formação dos cachos, pois a partir desta fase fenológica da cultura intensifica-se a divisão de fotoassimilados entre o sistema vegetativo e reprodutivo, onde as plantas com maior disponibilidade hídrica se sobressaem na alocação destes para ambos os sistemas.

Isto pode ser observado na Figura 1 que apresenta a variação da altura de plantas em função dos níveis de tensão utilizados, evidenciando a predominância do tratamento irrigado com a tensão de $15 \mathrm{kPa}$ que recebeu a maior lâmina de água.

Figura 1. Altura de plantas da mamoneira em função da irrigação a diferentes tensões de água no solo
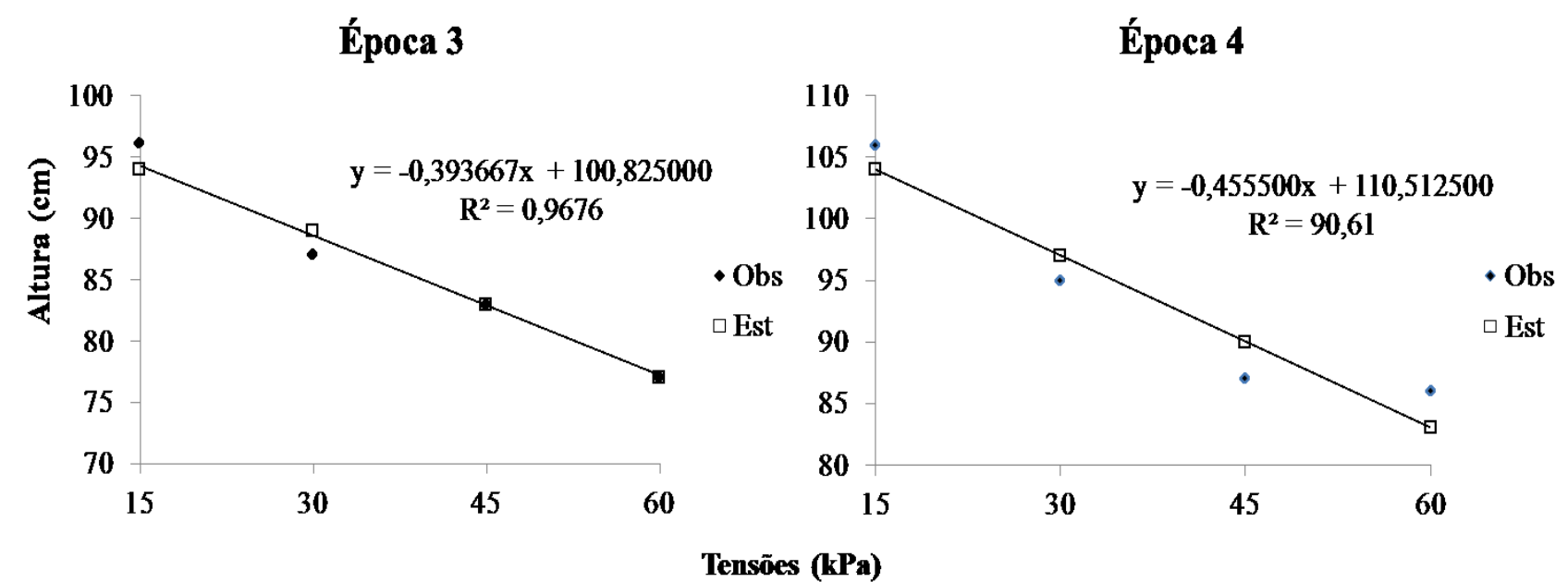

$\mathrm{Na}$ época 3 o tratamento $\mathrm{T} 1$ apresentou a maior média de altura com crescimento 11,$84 ; 17,04$ e $24,68 \%$ superior aos tratamentos T2, T3 e T4, respectivamente. $\mathrm{Na}$ época 4 o tratamento T1 manteve a superioridade, com altura média 14,$16 ; 24,55$ e $25,84 \%$ superior aos tratamentos $\mathrm{T} 2, \mathrm{~T} 3$ e $\mathrm{T} 4$, respectivamente. Estes resultados indicam que o manejo de irrigação realizado com menores flutuações dos níveis de tensão de água no solo favorecem o crescimento da mamoneira.

Resultado semelhante também foi observado por Souza et al. (2007) que variaram a época de semeadura entre estação seca e chuvosa no estado do Ceará com irrigação suplementar, obtendo maior 
altura de plantas para os tratamentos que receberam maior lâmina de água.

Na Tabela 4 apresenta-se a análise de variância para as características relacionadas à produção da mamoneira, onde observou-se diferença significativa ao nível de $5 \%$ de probabilidade pelo teste de "F" para o peso de sementes com baga e para o peso de sementes sem baga em relação ao manejo da irrigação feito com base nos diferentes níveis de tensão de água no solo.

Tabela 4. Resumo da análise de variância para as características peso de sementes com baga e peso de sementes sem baga ( $\mathrm{g}$ ) de mamoneira irrigada sob diferentes tensões, em Lavras-MG, 2010 .

\begin{tabular}{lccc}
\hline \multirow{2}{*}{ FV } & GL & \multicolumn{2}{c}{ Quadrados Médios } \\
\cline { 3 - 4 } & & $\begin{array}{c}\text { Peso com } \\
\text { baga }\end{array}$ & $\begin{array}{c}\text { Peso sem } \\
\text { baga }\end{array}$ \\
\hline Tensões $(\mathrm{T})$ & 3 & $179255,73^{* *}$ & $75835,42^{* *}$ \\
Blocos & 3 & $171447,39^{*}$ & $59552,08^{*}$ \\
Erro & 9 & 46091,84 & 16236,81 \\
\hline CV(\%) & & 20,00 & 20,09 \\
\hline Média & & 1073,4 & 634,4 \\
\hline
\end{tabular}

ns - não significativo, $* * *$ significativo a $1 \%, * *$ significativo a $5 \%$, ${ }^{*}$ significativo a $10 \%$ de probabilidade, pelo teste $\mathrm{F}$.

Nas Figuras 2A e 2B pode-se observar redução de forma linear para produção de sementes com baga e sem baga à medida que se aumentou a tensão de água no solo. Tal fato demonstra que o manejo da irrigação com tensões menores do que 15
$\mathrm{kPa}$ possivelmente promoveriam maiores acréscimos de produtividade, pois até esse nível de tensão, não houve indício de que a cultura tenha sofrido algum estresse por anoxia.

Figura 2. Peso das sementes com baga (A.) e peso das sementes sem baga (B.) em função das tensões de água no solo
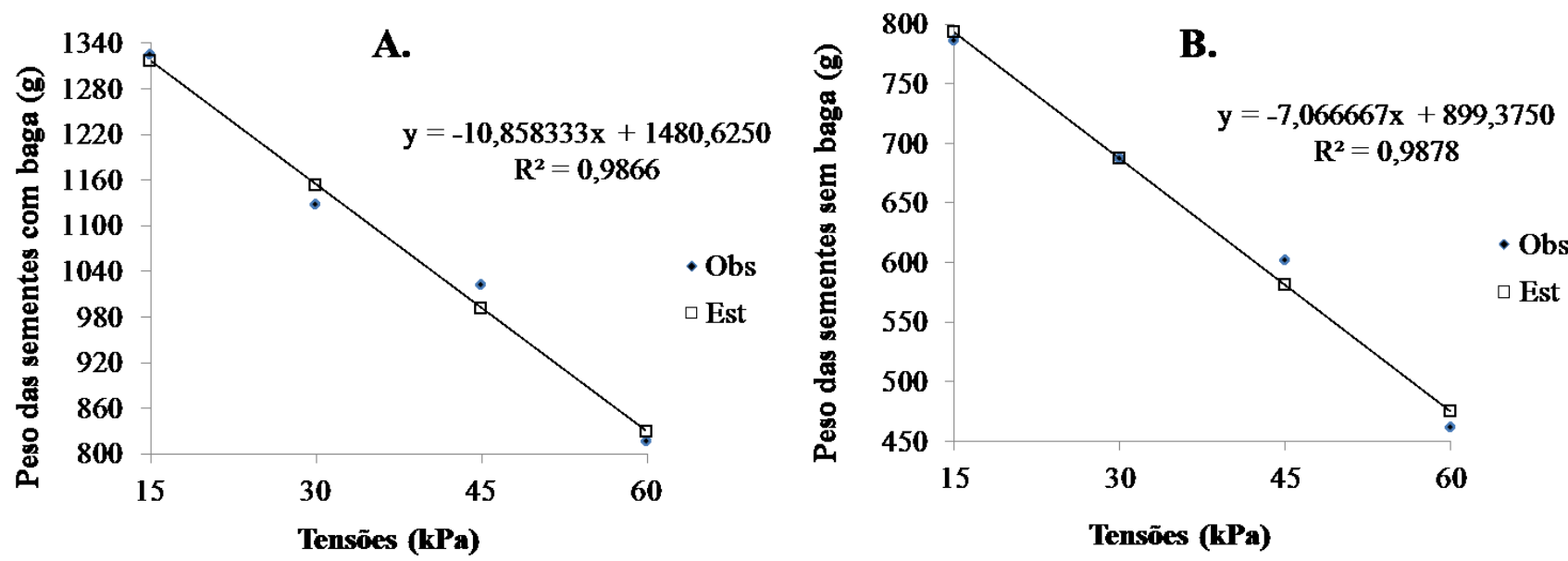
Ao se realizar os cálculos para obtenção da produtividade $\left(\mathrm{kg} \mathrm{ha}{ }^{-1}\right)$ de sementes sem baga de cada tratamento, observou-se valores de 4.700, 4.100, 3.600, 2.750 e $460 \mathrm{~kg} \mathrm{ha}^{-1}$, respectivamente, para os tratamentos T1 $(15 \mathrm{kPa}), \mathrm{T} 2(30 \mathrm{kPa}), \mathrm{T} 3$ (45 kPa), T4 $(60 \mathrm{kPa})$ e para a testemunha sem irrigação.

Estes resultados são semelhantes aos observados por Viana et al. (2009) que obtiveram produtividades crescentes para a mamoneira, variedade IAC Guarani, em patamares semelhantes, de 2.600, 3.450, 4.050 e $4.161 \mathrm{~kg} \mathrm{ha}^{-1}$, respectivamente, para os níveis de irrigação equivalentes a 25, 50, 75 e $100 \%$ da evaporação do tanque reduzido.

Igualmente, Rios et al. (2014), estudando o efeito de diferentes tensões de água no solo no cultivo da mamoneira, variedade Al Guarany 2002, observaram que a maior produtividade, de $1.994,14 \mathrm{~kg}$ $\mathrm{ha}^{-1}$, foi obtida no tratamento irrigado com o valor de tensão de $15 \mathrm{kPa}$ e a menor produtividade foi de $773,91 \mathrm{~kg} \mathrm{ha}^{-1}$, no tratamento irrigado na tensão de $75 \mathrm{kPa}$.

Como o experimento dos referidos autores também foi conduzido em Lavras MG na mesma época do ano, coincidindo com a estação seca, mas utilizando um espaçamento de plantio de 2,4 $\mathrm{m}$ entre linhas e $0,75 \mathrm{~m}$ entre plantas na linha, depreende-se que a diferença de produtividade, de cerca de $2700 \mathrm{~kg} \mathrm{ha}^{-1}$, se deva ao adensamento de plantio proposto pelo presente trabalho.

\section{CONCLUSÕES}

$\mathrm{O}$ adensamento do plantio da cultura da mamoneira interfere no crescimento da mesma.

A mamoneira cultivada em plantio adensado apresenta incremento positivo de produtividade à medida que se aumenta a lâmina de irrigação.

A maior produtividade para a mamoneira cultivada sob irrigação em plantio adensado foi de $4.700 \mathrm{~kg} \mathrm{ha}^{-1}$ com o valor de tensão de água no solo de $15 \mathrm{kPa}$.

\section{AGRADECIMENTOS}

À CAPES, pela concessão de bolsa de estudos. Ao CNPq, à FAPEMIG e à FINEP, pelo apoio aos projetos envolvendo plantas oleaginosas que têm sido desenvolvidos na UFLA.

\section{REFERÊNCIAS}

AMARAL, J. G. C. (2002) Mamona Al

Guarany 2002. Campinas: CATI, 2002.

Disponível em: <

http://www.cati.sp.gov.br/portal/produtose-servicos/publicacoes/acervotecnico/mamona-al-guarany-2002 >. Acesso em: 24 set. 2017.

BRASIL. Ministério da Agricultura, Pecuária e Abastecimento. Mamona.

Campina Grande: Embrapa Algodão, 2010. Disponível em:

$<$ http://www.cnpa.embrapa.br/produtos/ma 
mona/ index.html $>$. Acesso em: 24 set. 2017.

BARROS JÚNIOR, G. et al. Consumo de água e eficiência do uso para duas cultivares de mamona submetidas a estresse hídrico. Revista Brasileira de Engenharia Agrícola e Ambiental, v.12, p.350-355, 2008.

BELTRÃO, N. E. M. Sistema de produção de mamona em condições irrigadas: considerações Gerais. Campina Grande: Embrapa Algodão. 14 p. (Documentos, 132), 2004.

CARVALHO, B. C. L. de. Manual do cultivo da mamona. Salvador: EBDA. 65 p., 2005.

CARVALHO, E. V. de. et al. Densidade de plantio em duas cultivares de mamona no sul do Tocantins. Revista Ciência Agronômica, v.41, p.387-392, 2010.

DANTAS, A. A. A.; CARVALHO, L. G.; FERREIRA, E. Classificação e tendências climáticas em Lavras, MG. Ciência e Agrotecnologia, v.31, p.1862-1866, 2007.

DOURADO NETO, D.; VAN LIER, Q. J.; BOTREL, T. A. Programa para confecção da curva de retenção da água no solo utilizando modelo Genuchten. Engenharia Rural, v.1, p.92-102, 1990.

EMPRESA BRASILEIRA DE PESQUISA AGROPECUÁRIA. Centro Nacional de Pesquisas de Solos. Sistema brasileiro de classificação de solos. 2. ed. Rio de Janeiro: Embrapa Solos. 306 p, 2006.

FERREIRA, D. F. SISVAR: a computer statistical analysis system. Ciência e Agrotecnologia, Lavras, v.35, n.6, p.10391042, 2011.

FREITAS, C. A. S. de. et al. Comportamento de cultivares de mamona em níveis de irrigação por gotejamento em
Pentecoste, CE. Revista Brasileira de Engenharia Agrícola e Ambiental, v.14, p.1059-1066, 2010.

GUIMARÃES, P. T. G. et al. Mamoneira. In: RIBEIRO, A. C.; GUIMARÃES, P. T. G.; VENEGAS, V. H. A. (Ed.).

Recomendação para o uso de corretivos e fertilizantes em Minas Gerais: $5^{\circ}$

Aproximação. Viçosa: CFSEMG, p.289302, 1999.

LIMA, E. P.; SILVA, E. L. Temperatura base, coeficientes de cultura e graus-dia para cafeeiro arábica em fase de implantação. Revista Brasileira de Engenharia Agrícola e Ambiental, v.12, p.266-273, 2008.

RIOS, G. F. A. et al. Tensões de água no solo no cultivo de mamoneira. Pesquisa Agropecuária Tropical, v.44, n.3, p. 287293, 2014.

RODRIGUES, L. N. et al. Crescimento e produção de bagas da mamoneira irrigada com água residuárias doméstica. Revista Brasileira de Engenharia Agrícola e Ambiental, v.13, p.825-835, 2009.

SAVY FILHO, A. et al. IAC-2028: nova cultivar de mamona. Pesquisa

Agropecuária Brasileira, v.42, p.449452, 2007.

SEVERINO, L. S. et al. Crescimento e produtividade da mamoneira influenciada por plantio em diferentes espaçamentos entre linhas. Revista Ciência Agronômica, v.37, p.50-54, 2006.

SILVA, S. M. S. et al. Dotações hídricas em densidades de plantas na cultura da mamoneira cv. BRS Energia. Revista Brasileira de Ciências Agrárias, v.4, n.3, p. 338-348, 2009.

SORATTO, R. P. et al. Espaçamento e população de plantas de mamoneira de porte baixo para colheita mecanizada. 
Pesquisa Agropecuária Brasileira, v.46, p.245-253, 2011.

SOUZA, A. dos S. et al. Épocas de plantio e manejo da irrigação para a mamoneira. II - crescimento e produtividade. Revista Ciência Agronômica, v.38, p.422-429, 2007.

SOUZA-SCHLICK, G. D. de. et al. Crescimento e produtividade da mamona de porte baixo em diferentes espaçamentos e populações de plantas. Interciencia, v.37, p.49-54, 2012.

SOUZA-SCHLICK, G. D. de. et al. Desempenho da mamoneira IAC $2028 \mathrm{em}$ função do espaçamento entre fileiras e população de plantas na safrinha.

Bragantia, v.70, p.519-528, 2011.

VAN GENUCHTEN, M. T. A closed-from equation for predicting the hydraulic conductivity of unsaturated soils. Soil Science Society of American Journal, Madison, v.44, n.5, p.892-898, 1980.

VIANA, T. V. de A. et al. Efeitos de níveis de irrigação, estimados a partir da evaporação medida no tanque reduzido, na cultura da mamoneira. Irriga, v.14, p.7687, 2009. 